\section{Neutralizing HIV rebound}

\section{By Benjamin Boettner, Associate Editor}

Although antiretroviral therapies have transformed HIV infection into a chronically manageable disease, they have made little impact in reaching the latent viral reservoirs that are sources of disease rebound. Researchers at The Rockefeller University have found that broadly neutralizing antibodies against the HIV envelope protein, when given with viral inducers, can purge the reservoirs of virus and potentially eliminate the virus from its last bastion. ${ }^{1}$

In a second study from Anthony Fauci's lab at the NIH's National Institute of Allergy and Infectious Diseases (NIAID), broadly neutralizing antibodies (bNAbs) suppressed latent HIV in vitro in T cells derived from patients treated with standard antiretroviral therapy (ART). ${ }^{2}$ Fauci is director of the NIAID.

ART inhibits HIV replication and suppresses viral load in patients. However, it is not curative, and latent virus particles inevitably start replicating over time if patients stop taking the drugs.

Previous attempts to knock out the viral reservoir included a 'shock-and-kill' approach that used inducers of RNA transcription to activate the virus and ART to clamp down on new virus particles as they formed. But that strategy has not worked convincingly, and researchers have begun to look at passive immunization with HIV bNAbs as a way to attack the viral reservoirs.

bNAbs arise in about $20 \%$ of HIV cases and are detected about two years after initial infection. They bind epitopes on the HIV envelope proteins and can prevent fusion of viral and target cell membranes, thus blocking infection of $\mathrm{CD} 4^{+} \mathrm{T}$ cells.

Michel Nussenzweig, principal investigator in the Rockefeller University group, previously found the first clues that bNAbs could suppress latent virus when his lab studied HIV in primates. In a pair of 2013 papers, the group showed for the first time that bNAbs can damp down viral rebound and showed that the antibodies can induce passive immunity in a macaque model of SHIV-a hybrid virus derived from HIV and simian immunodeficiency virus (SIV). ${ }^{3,4}$

Now, Nussenzweig has used a humanized mouse model of HIV infection to show that bNAbs can activate the silent reservoir and that the antibodies use their $\mathrm{Fc}$ domains to recruit the immune system to help eliminate the virus. Nussenzweig is a professor at Rockefeller University and a Howard Hughes Medical Institute investigator.

In the NIAID study, Fauci's group studied HIV released from latent reservoirs and found that specific bNAbs can prevent the virus from replicating and infecting $\mathrm{CD}^{+} \mathrm{T}$ cells in vitro. The data suggest bNAbs could prevent viral rebound in patients who stop taking ART.

Fauci told SciBX, "The elegant study by Nussenzweig and colleagues in Cell provides an approach that is aimed more at elimination of the reservoir, whereas our future studies will be directed more at chronic suppression of the reservoir by intermittent administration of antibodies. The common denominator of both studies is the important role that bNAbs could play in the suppression or elimination of viral reservoirs in the absence of antiretroviral therapy."

\section{Reservoir jogs}

The Rockefeller University study used a mix of bNAbs isolated from patients with HIV-neutralizing serum and showed that the antibodies delayed viral rebound versus ART when given to mice with established infection. The experiment involved giving bNAbs or ART four days after infecting humanized mice with HIV and then following viral rebound in the plasma after therapy was terminated.

Whereas 18 of 22 mice given ART displayed viremia 28-84 days after the last injection, only 10 out of 21 bNAb-treated mice developed viremia, and in 9 of those it took 74-101 days to appear.

Next, the team showed that bNAbs required their $\mathrm{Fc}$ domains to produce the effect. Mutant bNAbs that could not interact with $\mathrm{Fc}$ receptors were much less efficient at suppressing viral rebound than the wild-type antibodies. For the mutant antibodies, 9 of 15 mice developed viremia within 44 days versus 1 of 21 mice given wild-type bNAbs.

The results suggested that a broad immune response was initiated by the bNAbs and that Fc receptor-expressing immune cells enhanced the antibodies' effect.

To test whether there was a more efficient way of emptying the reservoirs, the team borrowed from the 'shock-and-kill' playbook and combined the bNAbs with agents that induce viral transcription in latently infected cells.

In bNAb-treated, humanized mice with undetectable levels of virus, a combination of three viral inducers prevented viral rebound in $57 \%$ of the mice, whereas single inducer administration or no inducer treatment prevented viral rebound in only $10 \%$ of mice.

The viral inducers used were the histone deacetylase (HDAC) inhibitor Zolinza vorinostat, a BET bromodomain protein inhibitor and an immune-modulatory anti-CTLA-4 (CD152) antibody. Vorinostat is marketed by Merck \& Co. Inc. for cutaneous T cell lymphoma (CTCL) and is in clinical development for other cancers. The BET inhibitor was obtained from GlaxoSmithKline plc's 
BET inhibitor program. The program's most advanced compound is GSK525762, which is in Phase I trials for epithelial cancer. Nussenzweig did not disclose the specific CTLA-4 antibody used.

Results were published in Nature.

In the second study, the NIAID team used latently infected CD $4^{+}$ $\mathrm{T}$ cells derived from patients treated with ART to screen for antibodies that could suppress the viral reservoir.

The researchers provoked the isolated $\mathrm{CD}^{+} \mathrm{T}$ cells into releasing intact and infectious virions into culture using anti-CD3 and anti-CD28 antibodies and then assayed a panel of bNAbs for their ability to bind the virus particles. They identified a subset of bNAbs that prevented viral entry into $\mathrm{CD} 4^{+} \mathrm{T}$ cells from uninfected individuals.

The bNAbs' ability to inhibit HIV entry suggested they could block reinfection of T cells by HIV released from latent reservoirs.

Finally, the team showed that the bNAbs also suppressed replication of HIV released from infected, latent $\mathrm{CD} 4^{+} \mathrm{T}$ cells. They noted a correlation between the bNAbs' suppression of viral replication and their ability to inhibit viral entry. Thus, the researchers concluded that the bNAbs they tested could suppress the HIV viral reservoir in CD4 ${ }^{+}$ T cells after ART treatment has been stopped.

The study was published in the Proceedings of the National Academy of Sciences.

\section{Optimizing combos}

Although the bNAbs in both studies demonstrated proof of principle for suppressing HIV rebound, the researchers might need to optimize the antibodies and the combination with viral inducers. Two goals, they said, are to learn why $43 \%$ of the mice did show viral rebound and to improve the therapy's success rate.

Understanding the mechanism driving latency and the effect of viral inducers will be key to moving forward, said Nussenzweig. "We need to find out where the latent viruses are integrated in the genome, why they are latent and how the latent cell reservoir is composed in its totality.”

Matthew Marsden, an assistant researcher in the Department of Medicine and AIDS Institute of the University of California, Los Angeles, also emphasized the importance of the mechanism.

"For therapeutic developments, it will be interesting to evaluate exactly how the bNAbs function to reduce the number of HIV reservoir cells in the humanized mice and to define how much HIV expression is required for the effect. Some latency-reversing agents only induce low levels of HIV expression, and it will be useful to determine whether this low level of HIV protein expression is sufficient to make the cells susceptible to clearance mediated through bNAbs."

According to Mark Connors, chief of the HIV-specific immunity section at the NIAID, selecting different antibodies could produce better effects. "Although the bNAb combination employed by the Nussenzweig study worked well at eliminating the reservoir, new antibodies with non-overlapping specificities could further help increase HIV neutralization and prevent the risk of viral escape," he said.

Connors' lab has identified an antibody-dubbed 35O22-that has potent neutralizing activity against a range of HIV strains and that binds to a new epitope of the envelope proteins not recognized by other bNAbs characterized so far. ${ }^{5}$ The NIH has filed a provisional patent on $35 \mathrm{O} 22$, and the IP is available for licensing.
Dennis Burton said that future efforts should focus on two aspects of engineering Fc regions: enhancing binding efficiency of bNAbs both to leukocyte $F c$ receptors to improve effector function and to the neonatal Fc receptor, FcRn, to improve antibody half-life. "Recently, it was shown that affinity for the FcRn improves protection of bNAbs in the SHIV model by extending antibody half-life," he added. ${ }^{6}$

Burton is a professor of immunology and microbial science at The Scripps Research Institute and specializes in HIV vaccine development based on information deduced from bNAb specificities.

\section{Model monkeys}

Although the results in mice suggest bNAbs might help prevent viral rebound, nonhuman primates represent a more reliable model. Humanized mice allow infection of human cells with authentic HIV strains, but the mice do not acquire all the immune cells involved in HIV infection. In addition, the human grafts in mice only survive for a limited time.

The macaque model, on the other hand, is an immunologically intact host. However, it relies on infection with SHIV, whose evoked responses still differ from those in humans infected with HIV.

Marsden told SciBX, “To achieve the final goal of purging latent reservoirs of HIV in human patients, the most straightforward next translational step would be to test latency-reversing agents and bNAbs in a suitable nonhuman primate model."

Burton agreed. "There are activators that could be used in macaques, and the antibodies are compatible with SHIV infection. Those viruses are not exactly the same, but they do cause AIDS and establish latency," he said.

However, he added that the viral reservoir could differ between humans and both primates and mice. "HIV reservoirs in the brain could pose a specific problem from the perspective of drug targeting," he said.

According to Marsden, "Clinical testing using the bNAbs alone to reduce viral loads as an alternative or complement to ART should not be too challenging; however, the new combinations of viral inducers and bNAbs are more complex and would thus likely require more comprehensive testing in preclinical models before any human trials."

Burton said that a clinical study could involve taking patients off ART and treating them with an antibody combination while carefully monitoring viral rebound.

Nussenzweig told SciBX that his team has started a Phase I trial in infected individuals with a single bNAb but did not disclose details of the trial design. He is planning a separate trial to test the antibodies together with viral inducers.

A patent has been filed for the use of the bNAb combination for HIV rebound therapy by Rockefeller University, and the IP is available for licensing. The findings from the NIH study have not been patented.

Boettner, B. SciBX 7(36); doi:10.1038/scibx.2014.1059

Published online Sept. 18, 2014

\section{REFERENCES}

1. Halper-Stromberg, A. et al. Cell; published online Aug. 14, 2014; doi:10.1016/j.cell.2014.07.043

Contact: Michel C. Nussenzweig, The Rockefeller University, New York, N.Y.

e-mail: nussen@rockefeller.edu 
2. Chun, T.-W. et al. Proc. Natl. Acad. Sci. USA; published online Aug. 25, 2014; doi:10.1073/pnas.1414148111

Contact: Anthony S. Fauci, National Institute of Allergy and Infectious Diseases, Bethesda, Md.

e-mail: afauci@niaid.nih.gov

Contact: Tae-Wook Chun, same affiliation as above e-mail: twchun@nih.gov

3. Barouch, D.H. et al. Nature 503, 224-229 (2013)

4. Shingai, M. et al. Nature 503, 277-281 (2013)

5. Huang, J. et al. Nature; published online Sep. 3, 2014; doi:10.1038/nature13601

6. Ko, S.-Y. et al. Nature; published online Aug. 13, 2014; doi:10.1038/nature13612
COMPANIES AND INSTITUTIONS MENTIONED

GlaxoSmithKline plc (LSE:GSK;NYSE:GSK), London, U.K.

Howard Hughes Medical Institute, Chevy Chase, Md.

Merck \& Co. Inc. (NYSE:MRK), Whitehouse Station, N.J.

National Institute of Allergy and Infectious Diseases, Bethesda, Md.

National Institutes of Health, Bethesda, Md.

The Rockefeller University, New York, N.Y.

The Scripps Research Institute, La Jolla, Calif.

University of California, Los Angeles, Calif. 